OPEN ACCESS

Edited by:

Jean-Pierre Saint-Jeannet,

New York University, United States

Reviewed by:

Sergei Sokol,

Icahn School of Medicine at

Mount Sinai, United States

Jean-Loup Duband,

INSERM U955 Institut Mondor

de Recherche Biomédicale (IMRB),

France

*Correspondence:

Marianne E. Bronner

mbronner@caltech.edu

Specialty section:

This article was submitted to Craniofacial Biology and Dental

Research,

a section of the journal

Frontiers in Physiology

Received: 30 October 2020

Accepted: 07 January 2021

Published: 03 February 2021

Citation:

Hutchins EJ, Piacentino ML and Bronner ME (2021) Transcriptomic Identification of Draxin-Responsive

Targets During Cranial Neural Crest EMT. Front. Physiol. 12:624037. doi: 10.3389/fphys.2021.624037

\section{Transcriptomic Identification of Draxin-Responsive Targets During Cranial Neural Crest EMT}

\author{
Erica J. Hutchins, Michael L. Piacentino and Marianne E. Bronner* \\ Division of Biology and Biological Engineering, California Institute of Technology, Pasadena, CA, United States
}

Canonical Wnt signaling plays an essential role in proper craniofacial morphogenesis, at least partially due to regulation of various aspects of cranial neural crest development. In an effort to gain insight into the etiology of craniofacial abnormalities resulting from Wnt signaling and/or cranial neural crest dysfunction, we sought to identify Wntresponsive targets during chick cranial neural crest development. To this end, we leveraged overexpression of a canonical Wnt antagonist, Draxin, in conjunction with RNA-sequencing of cranial neural crest cells that have just activated their epithelialmesenchymal transition (EMT) program. Through differential expression analysis, gene list functional annotation, hybridization chain reaction ( $\mathrm{HCR})$, and quantitative reverse transcription polymerase chain reaction (RT-qPCR), we validated a novel downstream target of canonical Wnt signaling in cranial neural crest - RHOB - and identified possible signaling pathway crosstalk underlying cranial neural crest migration. The results reveal novel putative targets of canonical Wnt signaling during cranial neural crest EMT and highlight important intersections across signaling pathways involved in craniofacial development.

Keywords: Draxin, Wnt, neural crest, EMT, craniofacial development

\section{INTRODUCTION}

The neural crest is a multipotent stem cell population in the vertebrate embryo that undergoes coordinated induction, specification, and epithelial-mesenchymal transition (EMT) events to migrate and ultimately differentiate into a wide range of cell types. The migratory pathways and derivatives formed by the neural crest are regionalized according to their axial level of origin, such that cells from a given axial level give rise to a characteristic array of progeny and follow distinct pathways from those arising at other axial levels (Gandhi and Bronner, 2018). The most anterior "cranial" neural crest population underlies much of the development of the face (Cordero et al., 2011), and is the only neural crest population in vivo with the ability to differentiate into facial skeleton, contributing to the upper and lower jaw, and bones of the neck (Noden, 1975; Le Douarin, 1982; Simoes-Costa and Bronner, 2015). Importantly, perturbation of various stages of cranial neural crest development results in a myriad of craniofacial malformations (Vega-Lopez et al., 2018).

Many facets of cranial neural crest development are regulated by Wnt signaling (Wu et al., 2003; Yanfeng et al., 2003; Steventon et al., 2009; Milet and Monsoro-Burq, 2012; Simoes-Costa et al., 2015; Rabadán et al., 2016; Hutchins and Bronner, 2018, 2019; Gandhi et al., 2020). Furthermore, 
Wnt signaling is critical for normal facial patterning; mutations in Wnt pathway components or dysregulation of canonical Wnt signaling output result in defects in craniofacial morphogenesis (Huelsken et al., 2000; Chiquet et al., 2008; Reid et al., 2011; He and Chen, 2012; Kurosaka et al., 2014). Thus, identification of canonical Wnt targets during cranial neural crest development would greatly enhance understanding the etiology of craniofacial abnormalities resulting from Wnt signaling or cranial neural crest dysfunction.

Here, we took advantage of a canonical Wnt signaling inhibitor, Draxin, to identify Wnt-responsive targets during chick cranial neural crest development. As Draxin overexpression impedes cranial neural crest EMT in a $\beta$-catenin-dependent mechanism (Hutchins and Bronner, 2018, 2019), here we utilize Draxin overexpression together with RNA-sequencing (RNAseq) on sorted populations of cranial neural crest cells to identify novel downstream targets of canonical Wnt signaling during cranial neural crest EMT.

\section{MATERIALS AND METHODS}

\section{Embryo Electroporation and Expression Constructs}

Electroporations were performed at Hamburger-Hamilton stage HH4 (Hamburger and Hamilton, 1951), using commercially available fertile chicken (Gallus gallus) eggs (Sunstate Ranch, Sylmar, CA, United States), as previously described (Hutchins and Bronner, 2018). The cranial neural crest-specific enhancer NC1.1m3:GFP (Simoes-Costa et al., 2012), Draxin overexpression (Hutchins and Bronner, 2018), BRE::GFP BMP reporter (Le Dreau et al., 2012), NC1- $\Delta 90 \beta$ cat canonical Wnt signaling activation (Hutchins and Bronner, 2018), and control expression (Betancur et al., 2010b) constructs were described previously.

\section{Tissue Dissociation and FACS}

Following electroporation, embryos were incubated at $37^{\circ} \mathrm{C}$ until $\mathrm{HH} 9+$. We then dissected embryonic heads anterior to the otic vesicle in Ringer's solution, washed tissue with sterile PBS (Corning cellgro \#21-031-CV), then incubated tissue in Accumax (Innovative Cell Technologies, Inc. \#AM-105) at $37^{\circ} \mathrm{C}$ for $15 \mathrm{~min}$, with trituration every $5 \mathrm{~min}$. Following dissociation, cells were washed with Hanks' Balanced Salt Solution (Thermo Fisher Scientific \#88284), filtered through a $20 \mu \mathrm{M}$ nylon net mesh filter (Millipore Product \#NY2004700), and resuspended in Hanks' supplemented with $0.25 \%$ bovine serum albumin and 5\% RQ1 DNase (Promega \#M6101). GFP + cells were then collected using fluorescence activated cell sorting (FACS) at the Caltech Flow Cytometry Cell Sorting Facility.

\section{Library Preparation and Sequencing}

We used $1500 \mathrm{GFP}+$ cranial neural crest cells per replicate to prepare libraries. cDNA libraries were prepared using the Takara Bio SMART-Seq v4 Ultra Low Input cDNA kit, according to manufacturer instructions. RNA-Seq was performed at the
Caltech Millard and Muriel Jacobs Genetics and Genomics Laboratory at 35 million reads on two biological replicates for both the control cranial and Draxin overexpression cranial neural crest cells. Sequencing libraries were built according to Illumina Standard Protocols and SR50 sequencing was performed in a HiSeq Illumina machine by the Caltech Millard and Muriel Jacobs Genetics and Genomics Laboratory. Sequence reads were aligned to the G. gallus genome (galgal6) with Bowtie2 (Langmead and Salzberg, 2012), transcript counts were calculated with HTSeq-Count (Anders et al., 2015), and differential expression analysis was performed with DESeq2 (Love et al., 2014). Gene lists were analyzed for functional annotation using PANTHER (Mi et al., 2019) and DAVID (Huang da et al., 2009a,b).

\section{Hybridization Chain Reaction}

Embryos to be processed for hybridization chain reaction (HCR) were fixed in $4 \%$ paraformaldehyde $1 \mathrm{~h}$ at room temperature, then dehydrated in graded methanol washes and stored at least one overnight at $-20^{\circ} \mathrm{C}$. HCR was performed as previously described (Gandhi et al., 2020), with custom probes designed and ordered through Molecular Technologies.

\section{Image Acquisition and Analysis}

Confocal images were acquired with an upright Zeiss LSM 880 at the Caltech Biological Imaging Facility. Images were minimally processed for brightness/contrast and pseudocolored using Fiji (Schindelin et al., 2012) and Adobe Photoshop 2020. Relative fluorescence intensity was determined in Fiji. For each whole mount image, the line tool was used to draw an ROI surrounding the area of neural crest indicated by positive HCR fluorescence for neural crest marker TFAP2 $\beta$. Integrated density measurements were quantified for ROIs on the control electroporated (left) and experimental electroporated (right) sides from the same embryo. Relative fluorescence intensity was then calculated by dividing the integrated density measurements for the experimental versus the control side of the same embryo. Statistical analyses were performed using Prism (8; GraphPad Software). $P$ values are defined in the text, and significance was established with $P<0.05$. $P$ values were calculated for embryos using one-tailed paired $t$-tests with integrated density measurements for control versus experimental sides, and for qRT-PCR using two-tailed one sample $t$-tests for $\Delta \Delta \mathrm{C}_{T}$ values.

\section{Quantitative Reverse Transcription PCR (RT-qPCR)}

RNA was extracted from sorted cells (Draxin overexpression) and dissected $\mathrm{HH} 9+$ embryonic half heads (NC1- $\Delta 90 \beta$ cat canonical Wnt signaling activation) using the RNAqueousMicro Total RNA Isolation Kit (Invitrogen), according to manufacturer instructions. Following RNA isolation in elution buffer, cDNA was reverse transcribed using the SuperScriptIII First-Strand Synthesis System (Invitrogen) with oligo-dT priming. Quantitative PCR (qPCR) was performed using gene-specific primers with FastStart Universal SYBR Green Master Mix with Rox (Roche) and cDNA (diluted 1:10) on a 
QuantStudio 3 Real-Time PCR System (Applied Biosystems) in triplicate. We determined $\Delta \mathrm{C}_{T}$ with normalization against $18 \mathrm{~S}$ ribosomal RNA $\left(\Delta \mathrm{C}_{T}=\right.$ Target $\left.\mathrm{C}_{T}-18 \mathrm{~S} \mathrm{C}_{T}\right)$ for Draxin, $S N A I 2$, and $R H O B$ for samples, then calculated $\Delta \Delta C_{T}$ values $\left(\Delta \Delta \mathrm{C}_{T}=\right.$ Average Control $\Delta \mathrm{C}_{T}-$ Perturbation $\Delta \mathrm{C}_{T}$ ) for each target and replicate. The gene-specific primers used for $\mathrm{qPCR}$ were: Draxin-F 5'-CTACGCTGTTATGCCAAATTCC; DraxinR 5'-GAATGATCCCTGCTCTCCATT; SNAI2-F 5'-GCA ACAAGACCTATTCCACTTTC; SNAI2-R 5'-GTACTTG CAGCTGAACGATTTC; RHOB-F 5'-CGTGATCCTCATGT GCTTCT; RHOB-R 5'-TGCGCAGGTCTTTCTTGT; 18S-F 5'-CCATGATTAAGAGGGACGGC; 18S-R 5'-TGGCAAA TGCTTTCGCTTT.

\section{RESULTS}

\section{Identification of Draxin-Responsive Genes in Migrating Cranial Neural Crest}

We have previously shown that the secreted protein Draxin functions as a potent inhibitor of cranial neural crest cell migration during EMT (Figure 1A; Hutchins and Bronner, 2018, 2019). Its effects on neural crest are elicited extracellularly via $\beta$-catenin-dependent Wnt signaling inhibition, precisely at the early stages of cranial neural crest EMT at HH9+ (Hutchins and Bronner, 2018). To parse the cranial-specific targets of Draxin underlying its effect on neural crest EMT and uncover potential novel targets of canonical Wnt signaling, we performed RNA-sequencing (RNA-seq) on sorted chick cranial neural crest cells, with and without Draxin-mediated Wnt inhibition. To this end, we co-electroporated the FoxD3 NC1.1m3 enhancer, which drives GFP expression specifically in the cranial neural crest population (Simoes-Costa et al., 2012), with either a Draxin overexpression construct containing an internal ribosomal entry site (IRES) driving H2B-RFP (Hutchins and Bronner, 2018) or the same construct without the Draxin coding region as a control (Figure 1B). Embryos were subsequently developed to the onset of cranial neural crest EMT (HH9+), by which point Draxinmediated effects on EMT are evident (Figure 1C; Hutchins and Bronner, 2018, 2019), to identify EMT-related genes sensitive to canonical Wnt inhibition. From heads dissected anterior to the otic vesicle, we isolated $1500 \mathrm{GFP}+$ cranial neural crest cells per replicate by FACS, then performed $c D N A$ library preparation and sequencing (Figure 1D).

Differential expression analysis initially revealed 284 differentially expressed genes with $\geq 1.8$ fold change and FDR $<0.01$. For subsequent functional analysis, we filtered the gene lists to exclude lowly expressed genes (average normalized count values $<1000$ ), resulting in a filtered list of 134 differentially expressed genes (36 downregulated, 98 upregulated) (Figure 2A). Using PANTHER analysis (Mi et al., 2019) to probe molecular functions of these gene targets, we observed enrichment of factors highly associated with transcriptional regulation, enzymatic reactions (including kinases) and secreted proteins indicative of targets associated with intracellular signaling pathways, and structural molecules (such as cytoskeletal and extracellular matrix proteins) indicative of cell migration-associated targets (Figure 2B). Among the most highly changed genes, we found significant enrichment of Draxin, as expected due to its experimental overexpression. Interestingly, we also detected significant downregulation of the Notch pathway effector HES5 (and related genes), and BMP4 (as well as its downstream target MSX1) (Figures 2C,D), suggesting potential signaling pathway crosstalk between Draxin, canonical Wnt signaling, and other pathways with critical roles in neural crest development. Given that Draxin has been shown to intersect with additional signaling pathways in other contexts (Ahmed et al., 2011; Hossain et al., 2013; Meli et al., 2015), further studies are needed to parse direct and indirect effects relevant to craniofacial morphogenesis and neural crest EMT.

\section{Biological Pathway Analysis of Draxin-Responsive Genes in Cranial Neural Crest}

To better understand the molecular processes in which Draxin, and by extension canonical Wnt signaling, function during cranial neural crest EMT, we performed functional annotation for the dataset using the Database for Annotation, Visualization and Integrated Discovery (DAVID) (Huang da et al., 2009a,b). Consistent with established roles of canonical Wnt signaling and Draxin-mediated inhibition during cranial neural crest EMT, we observed enrichment of genes associated with transcriptional regulation, cell adhesion, and lipid synthesis, which we have recently shown is important for cell signaling during cranial neural crest EMT (Piacentino et al., 2020). In addition, we found numerous genes associated with bone/cartilage formation (e.g., CYTL1, ILK, NOV), a critical function of cranial neural crest, and genes involved in ribosome biogenesis (e.g., NOP56, PES1, NOC2L), which has implications for craniofacial development (Ross and Zarbalis, 2014) (Figure 3A).

Among the targets associated with transcriptional regulation, we detected significant downregulation of SNAI2, which has been shown to be a direct target of canonical Wnt signaling, and subsequently Draxin (LaBonne and Bronner-Fraser, 1998; Monsoro-Burq et al., 2005; Wu et al., 2005; Hutchins and Bronner, 2018) (Figure 3B). In addition, we observed significant downregulation of genes that mediate cell adhesion and EMT (Figure 3C), including $R H O B$, shown to be required for neural crest delamination in the trunk (Liu and Jessell, 1998), and EPCAM, which has been shown to participate in cancer cell EMT (Wang et al., 2018). We also observed significant correlations for disease-associated genes, including those involved in craniofacial (Figure 3D) and nervous system dysfunction (Figure 3E). This was expected given the role of the cranial neural crest in craniofacial development, and established roles for Draxin in nervous system development (Islam et al., 2009; Tawarayama et al., 2018).

\section{Functional Validation of a Novel Immediate Early Canonical Wnt Target}

To validate Draxin-responsive targets from our dataset, we performed quantitative $\mathrm{HCR}$ on embryos bilaterally electroporated with the Draxin overexpression construct on 

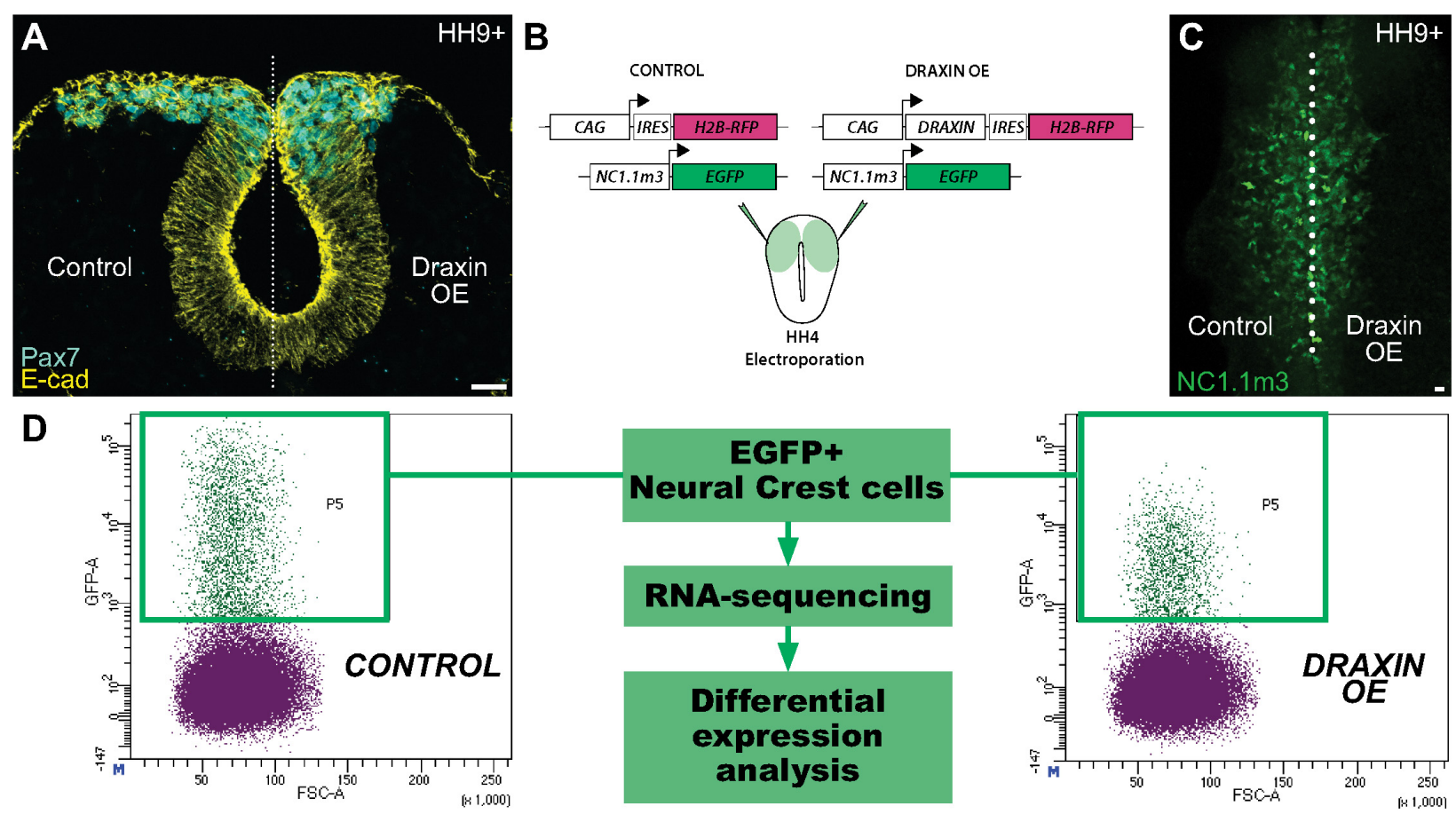

FIGURE 1 | Experimental design for identification of Draxin-responsive targets in cranial neural crest EMT. (A) Cross-section through embryo electroporated with a Draxin overexpression construct on the right side of the embryo. Immunostaining for E-cadherin (E-cad) and the neural crest marker Pax7 at HH9+ highlights the deleterious effects of Draxin overexpression on cranial neural crest EMT and migration away from the midline (dotted line), compared to the contralateral control side. (B-D) Experimental design to isolate cranial neural crest cells with or without Draxin overexpression. Gastrula stage chick embryos were co-electroporated with a neural crest-specific enhancer driving EGFP expression in cranial neural crest cells (NC1.1m3) and either a Draxin overexpression or control construct (B). NC1.1m3 enhancer expression revealed EGFP + cranial neural crest cells were responsive to Draxin overexpression and exhibited EMT defects (C). Fluorescence activated cell sorting (FACS) was used to isolate EGFP+ cranial neural crest cells with and without Draxin overexpression that were subsequently processed for RNA-sequencing and differential expression analysis (D). Scale bar, $20 \mu \mathrm{m}$. HH, Hamburger-Hamilton stage; OE, overexpression; IRES, internal ribosomal entry site.

the right side of embryos and the control construct on the left side (as in Figure 1B). To establish the area of neural crest migration from which to measure target fluorescence intensities, we visualized expression of TFAP2 $\beta$, a neural crest marker and non-target of Draxin. We focused on early HH9 + embryos, corresponding to the beginning of cranial neural crest EMT and initial stages of migration, in order to probe immediate early gene changes. As a result, modest defects were evident in the distance cranial neural crest cells migrated away from the midline (Figures 4A-D), consistent with a Draxin overexpression phenotype, albeit to a lesser extent than later stage $\mathrm{HH} 9+$ embryos in which migration has progressed more laterally (Figures 1A,C; Hutchins and Bronner, 2018, 2019). We measured SNAI2 and RHOB fluorescence intensities for Draxin overexpression versus control sides of individual embryos, and found significant downregulation of gene expression (Figure 4E; $78.0 \pm 2.8 \%$ of the control side for SNAI2 and $81.0 \pm 5.5 \%$ of the control side for RHOB; $P \leq 0.01$, one-tailed paired $t$-test), consistent with predicted trends based on our transcriptomic analyses. This is consistent with our previously published work indicating that Draxin acts upstream of Snail2 protein expression (Hutchins and Bronner, 2018). We further validated the effects of Draxin overexpression on SNAI2 and RHOB using quantitative reverse transcription PCR (RT-qPCR) with sorted cells collected alongside sequenced cells from Figure 1; consistent with the HCR data (Figures 4A-E), we detected significant downregulation of both $S N A I 2$ and $R H O B$ with Draxin overexpression (Figure $4 \mathbf{F}$ ).

Given that $R H O B$ has been previously shown to be a BMPresponsive target (Liu and Jessell, 1998) and insensitive to Wnt signaling (Taneyhill and Bronner-Fraser, 2005) in trunk neural crest, we next sought to determine whether the reduction in $R H O B$ we observed in cranial neural crest was due to direct effects from Wnt signaling, or indirect effects through downregulation of BMP. We have previously shown that canonical Wnt signaling is active in cranial neural crest at the onset of EMT using a fluorescent reporter (Hutchins and Bronner, 2018), while BMP signaling is active in the presumptive cranial neural crest at earlier stages during their induction (Piacentino and Bronner, 2018); here we employed a similar approach to investigate the timing of BMP signaling activation in cranial neural crest at the onset of EMT. Electroporation of a fluorescent BMP reporter (BRE::GFP) revealed a lack of active BMP signaling in cranial neural crest cells that have undergone EMT and commenced migration at HH9+ (Supplementary Figure 1). Thus, it is unlikely that the reduction in $R H O B$ we observed was due to suppressive effects on BMP signaling by Draxin. To more fully examine whether $R H O B$ downregulation was due to direct effects from Wnt signaling, we performed RT-qPCR on embryos with 


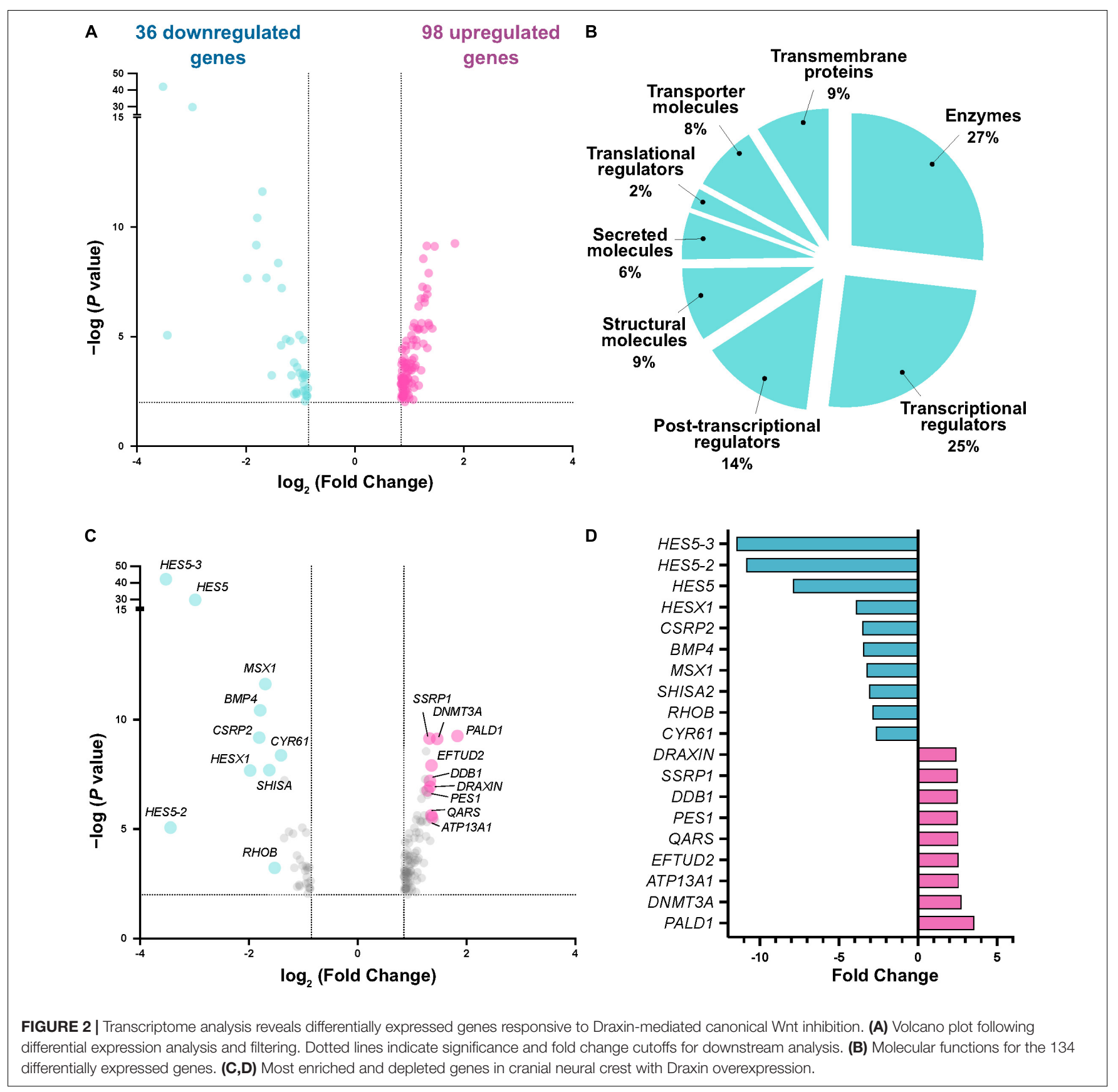

and without canonical Wnt signaling activation during cranial neural crest EMT. Here, we specifically activated canonical Wnt signaling in specified cranial neural crest by driving expression of a stabilized form of $\beta$-catenin under the control of a neural crestspecific enhancer (NC1- $\Delta 90 \beta$ cat). Importantly, we observed upregulation of SNAI2, an established direct target of canonical Wnt signaling (LaBonne and Bronner-Fraser, 1998; MonsoroBurq et al., 2005; Wu et al., 2005), as well as upregulation of $R H O B$, suggesting a direct link with Wnt signaling. Interestingly, we also observed concomitant downregulation of endogenous $D R A X I N$, suggesting the possibility of a negative feedback loop with respect to DRAXIN expression (Figure 4G).
Taken together, our data identify a novel target of Draxin and canonical Wnt signaling during cranial neural crest EMT (RHOB), and suggest that Draxin downregulation, and subsequent activation of Wnt signaling, is essential for crosstalk and feedback of signaling pathways that alter cranial neural crest transcriptional activation, and ultimately EMT.

\section{DISCUSSION}

Using transcriptome profiling of Draxin-responsive targets, we identified likely gene targets of canonical Wnt signaling 
A

Biological Pathways

Transcriptional Regulation (20)

$\begin{array}{llll}\text { MSX1 } & \text { CYTL1 } & \text { MYBL2 } & \text { SART3 } \\ \text { BMP4 } & \text { NOV } & \text { GATAD2A } & \text { AATF } \\ \text { SNAI2 } & \text { BTG2 } & \text { TCF3 } & \text { WWP2 } \\ \text { CYR61 } & \text { ETS1 } & \text { RICTOR } & \text { DNMT3A } \\ \text { EPCAM } & \text { SOX10 } & \text { NOC2L } & \text { HES5 }\end{array}$

Chondrocyte/Osteoblast

Differentiation (9)

Negative Regulation

of Apoptosis (8)

CYTL1 CYR61

NOV DDB1

BMP4 ILK

CYR61 EPCAM

HES5 MYBBP1A

BMP4 SOX10

NOV

PDLIM7

AATF

$M S X 1$

SNAI2

B

Transcriptional Regulation

C

Cell Adhesion (18)

HES5 FSCN1 TJP2 SH3GL1

CYR61 MFGE8 AATF SWAP70

EPCAM HDLBP IQGAP1 NOP56

HAPLN1 HAPLN3 MYH10

RHOB NRP2 NUDC

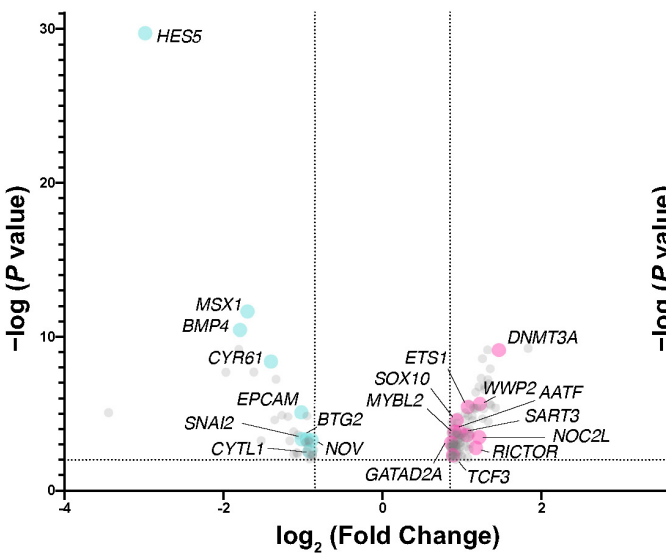

\begin{tabular}{|c|c|c|}
\hline \multicolumn{2}{|c|}{$\frac{\text { Ribosome }}{\text { Biogenesis (7) }}$} & $\begin{array}{c}\underline{\text { Lipid }} \\
\text { Synthesis (5) }\end{array}$ \\
\hline PES1 & UTP4 & $A C L Y$ \\
\hline WDR55 & NOL6 & HMGCS1 \\
\hline NOC2L & PDCD11 & FDPS \\
\hline NOP56 & & $\begin{array}{l}F D F T 1 \\
M V D\end{array}$ \\
\hline
\end{tabular}

Lipid

Synthesis (5)

$A C L Y$

HMGCS1

FDPS

FDFT1

$M V D$

Cell Adhesion

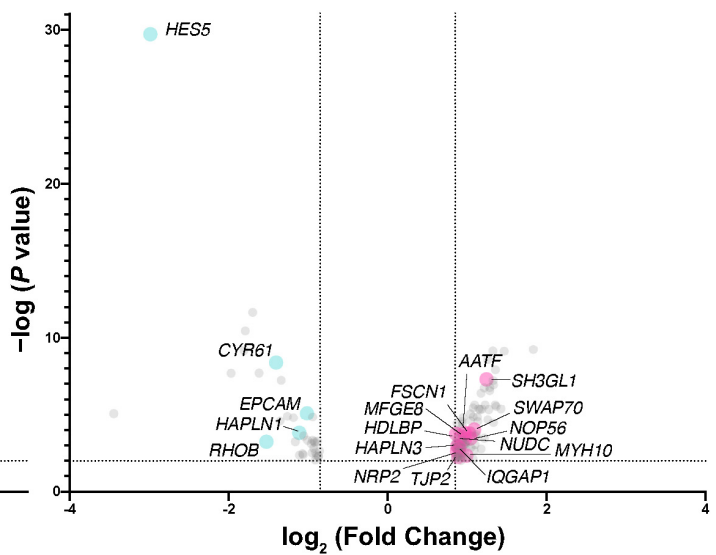

Disease Associations

D

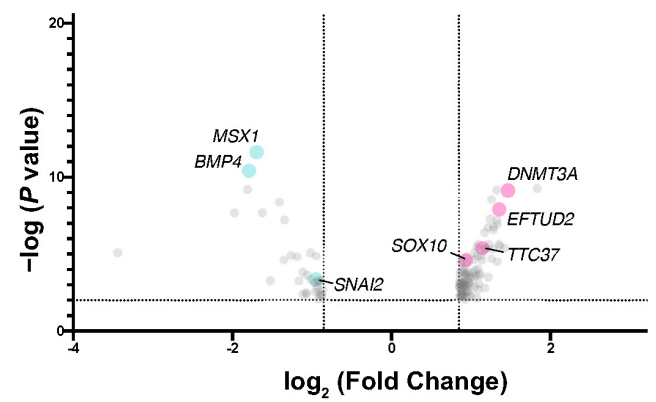

E

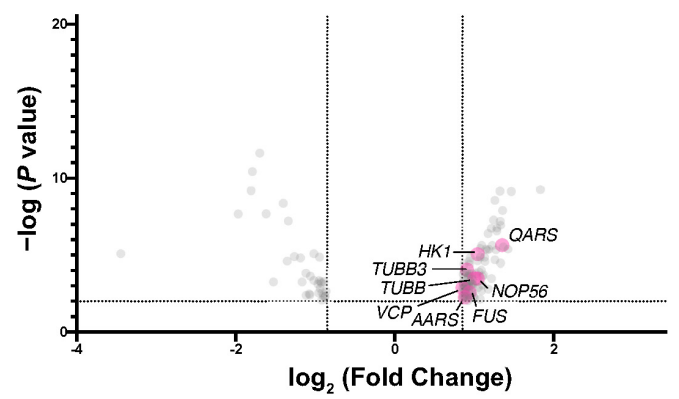

FIGURE 3 | Biological pathways and diseases associated with Draxin-responsive transcriptome dataset. (A-C) Biological pathway analysis identified enrichment of targets associated with transcriptional regulation, cell adhesion, chondrocyte/osteoblast differentiation, negative regulation of apoptosis, ribosome biogenesis, and lipid synthesis. (D,E) Functional annotation identified genes highly correlated with craniofacial abnormalities and nervous system dysfunction.

during cranial neural crest EMT. Consistent with our previously published work examining protein expression (Hutchins and Bronner, 2018), we verified transcript downregulation of canonical Wnt target SNAI2 in response to Draxin overexpression. Furthermore, we also identified and validated a novel target - RHOB. RhoB is BMP-responsive in trunk neural crest and is necessary for delamination (Liu and Jessell, 1998); its misexpression has been associated with defects in laminin organization within the basement membrane (Perez-Alcala et al., 2004). Interestingly, we have previously demonstrated that Draxin, via regulation of canonical Wnt signaling, also is involved in regulating laminin organization and 

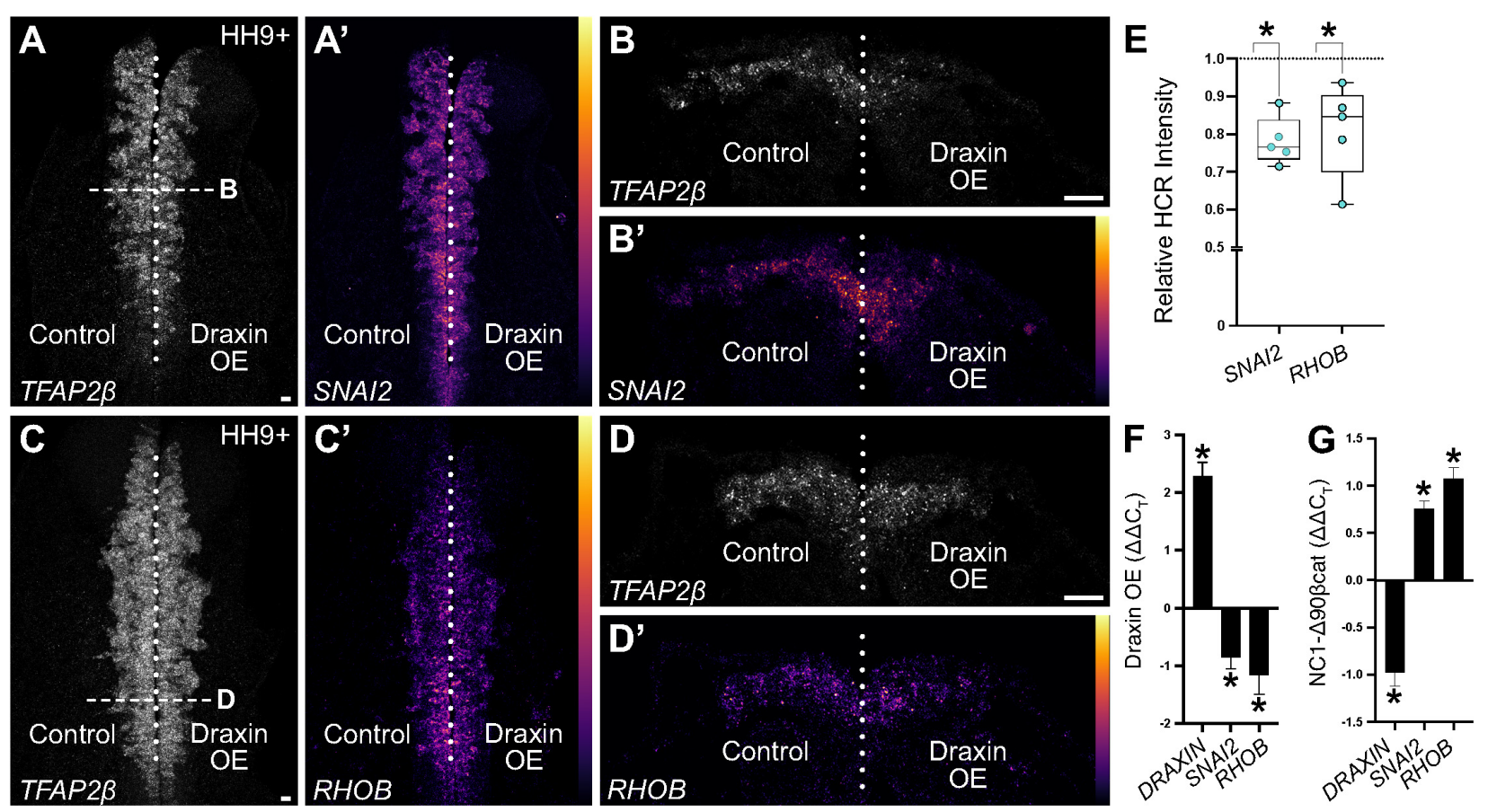

FIGURE 4 | Hybridization chain reaction (HCR) validation of identified neural crest EMT genes. (A-D) Representative images of quantitative HCR for neural crest marker TFAP2 $\beta$ and targets SNAI2 and RHOB in embryos bilaterally electroporated with a control (left) or Draxin overexpression (right) construct. SNAI2 and RHOB are pseudocolored based on fluorescence intensities as indicated by color scale in panels $\left(\mathbf{A}^{\prime}-\mathbf{D}^{\prime}\right)$. (E) Integrated density measurements revealed significant downregulation of SNAI2 and $R H O B(78.0 \pm 2.8 \%$ of the control side for SNAI2 and $81.0 \pm 5.5 \%$ of the control side for $R H O B$; $P \leq 0.01$, one-tailed paired $t$-test) with Draxin overexpression. Scale bar, $20 \mu \mathrm{m}$. (F) $\Delta \Delta \mathrm{C}_{T}$ values from quantitative reverse transcription PCR (RT-qPCR) for DRAXIN, SNAI2, and RHOB (normalized against 18S, comparing control versus overexpression cells) from HH9+ sorted cranial neural crest cells co-electroporated with NC1.1m3 fluorescent GFP reporter with or without Draxin overexpression (DraxinOE). As expected, DRAXIN was significantly upregulated whereas SNAI2 and RHOB were significantly downregulated in DraxinOE cranial neural crest cells. ${ }^{\star} P<0.05$, two-tailed one sample $t$-test. (G) $\Delta \Delta \mathrm{C}_{T}$ values from quantitative reverse transcription PCR (RT-qPCR) for $D R A X I N$, SNAI2, and RHOB (normalized against 18S, comparing control versus Wnt-activated cells) from dissected HH9+ cranial tissue from embryos co-electroporated with NC1.1m3 fluorescent RFP reporter with or without stabilized $\beta$-catenin expression (NC1- $\triangle 90 \beta$ cat). In contrast to Wnt inhibition via DraxinOE (F), SNAI2 and RHOB were significantly upregulated with Wnt activation via NC1- $\Delta 90 \beta$ cat, whereas $D R A X I N$ was significantly downregulated. ${ }^{\star} P \leq 0.02$, two-tailed one sample $t$-test.

remodeling of the basement membrane during cranial neural crest development (Hutchins and Bronner, 2019). Furthermore, we also observed downregulation of the BMP ligand BMP4, suggesting that BMP signaling may act downstream of Wnt signaling during or immediately after cranial neural crest EMT. In trunk, it has been shown that neural crest delamination is regulated by $\mathrm{BMP}$, and that canonical Wnt signaling is controlled by BMP signaling through BMP-responsive expression of the Wnt1 ligand (Burstyn-Cohen et al., 2004). This is particularly interesting in light of our observations from a GFP reporter construct that BMP signaling is inactive in cranial neural crest at the onset of EMT (Supplementary Figure 1). Interestingly, Draxin has also been shown to inhibit neural crest migration in the trunk ( $\mathrm{Su}$ et al., 2009; Zhang et al., 2017). Thus, whether RHOB expression is differentially regulated based on axial level (i.e., in response to BMP signaling in trunk neural crest versus in response to Wnt signaling in cranial neural crest) or based on signaling pathway crosstalk (which may also be dependent on axial level) remains to be explored.

In searching our datasets for neural crest-specific factors, we also noted modest upregulation of SOX10 and ETS1, genes associated with neural crest EMT (Tahtakran and Selleck, 2003; Theveneau et al., 2007; Simoes-Costa and Bronner, 2015), which seemed contradictory to the antagonistic role of Draxin in modulating cranial neural crest EMT. ETS1 expression is restricted to the cranial population of neural crest and is itself activated via cMYB (Betancur et al., 2010a); together with Sox9, Ets1 and cMYB function as activating gene regulatory inputs into a SOX10E2 enhancer (Betancur et al., 2010b), regulating SOX10 expression in cranial neural crest. Interestingly, in other contexts, canonical Wnt signaling has been shown to trigger degradation of cMYB protein (Kanei-Ishii et al., 2004); given that Draxin is endogenously expressed at HH9, it is possible that its normal inhibitory effects on canonical Wnt signaling may be necessary to reduce degradation of cMYB to activate endogenous levels of ETS1 and SOX10, which initiate expression prior to the onset of EMT. Thus, we postulate here that during early cranial neural crest migration, excess cMYB protein is stabilized via exogenous Draxin-mediated inhibition of canonical Wnt signaling; as a result, this may trigger upregulation of ETS1 and SOX10 gene expression. However, despite upregulation of factors positively associated with EMT, downregulation of SNAI2 alone is sufficient to 
impede cranial neural crest migration (Nieto et al., 1994; Hutchins and Bronner, 2019).

Taken together, our data identify novel targets of canonical Wnt signaling during cranial neural crest EMT, and highlight potential avenues of intersection for signaling pathways involved in craniofacial development. The results raise the intriguing possibility that the sequence and magnitude of signaling and gene expression crosstalk during cranial neural crest development may help precisely regulate craniofacial morphogenesis.

\section{DATA AVAILABILITY STATEMENT}

The datasets presented in this study can be found in online repositories. The names of the repository/repositories and accession number(s) can be found below: https://www.ncbi.nlm. nih.gov/bioproject/PRJNA673315.

\section{ETHICS STATEMENT}

Ethical review and approval was not required for the animal study because our study uses chicken embryos at E1-2. These are not considered vertebrate embryos until E10, and thus we do not require ethics committee approvals or protocols, as they are not considered vertebrates at the stages we work.

\section{AUTHOR CONTRIBUTIONS}

$\mathrm{EH}, \mathrm{MP}$, and $\mathrm{MB}$ conceived the project and conducted the experimental design and data interpretation. EH and MP performed the cell dissociations, library preparations, and RNA-seq analyses. EH performed the functional annotation, hybridization chain reaction experiments, imaging, quantitation, and statistical analyses. $\mathrm{EH}$ and $\mathrm{MB}$ wrote the manuscript

\section{REFERENCES}

Ahmed, G., Shinmyo, Y., Ohta, K., Islam, S. M., Hossain, M., Naser, I. B., et al. (2011). Draxin inhibits axonal outgrowth through the netrin receptor DCC. J. Neurosci. 31, 14018-14023. doi: 10.1523/JNEUROSCI.0943-11.2011

Anders, S., Pyl, P. T., and Huber, W. (2015). HTSeq-a Python framework to work with high-throughput sequencing data. Bioinformatics 31, 166-169. doi: 10.1093/bioinformatics/btu638

Betancur, P., Bronner-Fraser, M., and Sauka-Spengler, T. (2010a). Assembling neural crest regulatory circuits into a gene regulatory network. Annu. Rev. Cell Dev. Biol. 26, 581-603. doi: 10.1146/annurev.cellbio.042308.11 3245

Betancur, P., Bronner-Fraser, M., and Sauka-Spengler, T. (2010b). Genomic code for Sox10 activation reveals a key regulatory enhancer for cranial neural crest. Proc. Natl. Acad. Sci. U.S.A. 107, 3570-3575. doi: 10.1073/pnas.0906596107

Burstyn-Cohen, T., Stanleigh, J., Sela-Donenfeld, D., and Kalcheim, C. (2004). Canonical Wnt activity regulates trunk neural crest delamination linking $\mathrm{BMP} /$ noggin signaling with G1/S transition. Development $131,5327-5339$. doi: 10.1242/dev.01424

Chiquet, B. T., Blanton, S. H., Burt, A., Ma, D., Stal, S., Mulliken, J. B., et al. (2008). Variation in WNT genes is associated with non-syndromic cleft lip with or without cleft palate. Hum. Mol. Genet. 17, 2212-2218. doi: 10.1093/hmg/ ddn 121 with editing by MP. All authors contributed to the article and approved the submitted version.

\section{FUNDING}

This work was supported by the National Institutes of Health (R01DE027538 and R01DE027568 to MB, K99DE028592 to EH, and K99DE029240 to MP).

\section{ACKNOWLEDGMENTS}

We thank A. Collazo and G. Spigolon for imaging assistance at the Caltech Biological Imaging Facility; P. Cannon and R. Diamond of the Caltech Flow Cytometry Cell Sorting Facility for cell sorting assistance; I. Antoshechkin of the Caltech Millard and Muriel Jacobs Genetics and Genomics Laboratory for sequencing of our RNA-seq libraries; and M. Martik and S. Gandhi for assistance with data processing.

\section{SUPPLEMENTARY MATERIAL}

The Supplementary Material for this article can be found online at: https://www.frontiersin.org/articles/10.3389/fphys. 2021.624037/full\#supplementary-material

Supplementary Figure 1 | BMP-sensitive reporter expression is absent from delaminating and migratory cranial neural crest during EMT. (A) Schematic of cross section of cranial region at $\mathrm{HH} 9+$ examined in panel (B). Locations of premigratory $(\mathrm{pNC})$ and migratory crest cells $(\mathrm{mNC})$ are indicated. (B) Following electroporation of a BMP fluorescent reporter construct (BRE:GFP), cross sections stained for a neural crest marker (Snail2, magenta) and membrane label (WGA, white) revealed BMP reporter activity (green) in pNC (white dotted line), but not in delaminating or $\mathrm{mNC}$ (yellow dotted line). WGA, wheat germ agglutinin; pNC, premigratory neural crest; $\mathrm{mNC}$, migratory neural crest. Scale bar, $10 \mu \mathrm{m}$.

Cordero, D. R., Brugmann, S., Chu, Y., Bajpai, R., Jame, M., and Helms, J. A. (2011). Cranial neural crest cells on the move: their roles in craniofacial development. Am. J. Med. Genet. A 155A, 270-279. doi: 10.1002/ajmg.a.33702

Gandhi, S., and Bronner, M. E. (2018). Insights into neural crest development from studies of avian embryos. Int. J. Dev. Biol. 62, 183-194. doi: 10.1387/ijdb. $180038 \mathrm{sg}$

Gandhi, S., Hutchins, E. J., Maruszko, K., Park, J. H., Thomson, M., and Bronner, M. E. (2020). Bimodal function of chromatin remodeler Hmgal in neural crest induction and Wnt-dependent emigration. Elife 9:e57779. doi: 10.7554/eLife. 57779

Hamburger, V., and Hamilton, H. L. (1951). A series of normal stages in the development of the chick embryo. J. Morphol. 88, 49-92.

He, F., and Chen, Y. (2012). Wnt signaling in lip and palate development. Front. Oral Biol. 16:81-90. doi: 10.1159/000337619

Hossain, M., Ahmed, G., Naser, I. B., Shinmyo, Y., Ito, A., Riyadh, M. A., et al. (2013). The combinatorial guidance activities of draxin and Tsukushi are essential for forebrain commissure formation. Dev. Biol. 374, 58-70. doi: 10. 1016/j.ydbio.2012.11.029

Huang da, W., Sherman, B. T., and Lempicki, R. A. (2009a). Systematic and integrative analysis of large gene lists using DAVID bioinformatics resources. Nat. Protoc. 4, 44-57. doi: 10.1038/nprot.2008.211

Huang da, W., Sherman, B. T., Zheng, X., Yang, J., Imamichi, T., Stephens, R., et al. (2009b). Extracting biological meaning from large gene lists with DAVID. 
Curr. Protoc. Bioinformatics Chapter 13:Unit 13.11. doi: 10.1002/0471250953. bi1311s27

Huelsken, J., Vogel, R., Brinkmann, V., Erdmann, B., Birchmeier, C., and Birchmeier, W. (2000). Requirement for beta-catenin in anterior-posterior axis formation in mice. J. Cell Biol. 148, 567-578. doi: 10.1083/jcb.148.3.567

Hutchins, E. J., and Bronner, M. E. (2018). Draxin acts as a molecular rheostat of canonical Wnt signaling to control cranial neural crest EMT. J. Cell Biol. 217, 3683-3697. doi: $10.1083 /$ jcb.201709149

Hutchins, E. J., and Bronner, M. E. (2019). Draxin alters laminin organization during basement membrane remodeling to control cranial neural crest EMT. Dev. Biol. 446, 151-158. doi: 10.1016/j.ydbio.2018.12.021

Islam, S. M., Shinmyo, Y., Okafuji, T., Su, Y., Naser, I. B., Ahmed, G., et al. (2009). Draxin, a repulsive guidance protein for spinal cord and forebrain commissures. Science 323, 388-393. doi: 10.1126/science.1165187

Kanei-Ishii, C., Ninomiya-Tsuji, J., Tanikawa, J., Nomura, T., Ishitani, T., Kishida, S., et al. (2004). Wnt-1 signal induces phosphorylation and degradation of c-Myb protein via TAK1, HIPK2, and NLK. Genes Dev. 18, 816-829. doi: 10.1101/gad.1170604

Kurosaka, H., Iulianella, A., Williams, T., and Trainor, P. A. (2014). Disrupting hedgehog and WNT signaling interactions promotes cleft lip pathogenesis. J. Clin. Invest. 124, 1660-1671. doi: 10.1172/JCI72688

LaBonne, C., and Bronner-Fraser, M. (1998). Neural crest induction in Xenopus: evidence for a two-signal model. Development 125, 2403-2414.

Langmead, B., and Salzberg, S. L. (2012). Fast gapped-read alignment with Bowtie 2. Nat. Methods 9, 357-359. doi: 10.1038/nmeth.1923

Le Douarin, N. (1982). The Neural Crest. Cambridge: Cambridge University Press.

Le Dreau, G., Garcia-Campmany, L., Rabadan, M. A., Ferronha, T., Tozer, S., Briscoe, J., et al. (2012). Canonical BMP7 activity is required for the generation of discrete neuronal populations in the dorsal spinal cord. Development 139, 259-268. doi: $10.1242 / \mathrm{dev} .074948$

Liu, J. P., and Jessell, T. M. (1998). A role for rhoB in the delamination of neural crest cells from the dorsal neural tube. Development 125, 5055-5067.

Love, M. I., Huber, W., and Anders, S. (2014). Moderated estimation of fold change and dispersion for RNA-seq data with DESeq2. Genome Biol. 15:550. doi: 10.1186/s13059-014-0550-8

Meli, R., Weisova, P., and Propst, F. (2015). Repulsive axon guidance by Draxin is mediated by protein Kinase B (Akt), glycogen synthase kinase-3beta (GSK3beta) and microtubule-associated protein 1B. PLoS One 10:e0119524. doi: 10.1371/journal.pone.0119524

Mi, H., Muruganujan, A., Ebert, D., Huang, X., and Thomas, P. D. (2019). PANTHER version 14: more genomes, a new PANTHER GO-slim and improvements in enrichment analysis tools. Nucleic Acids Res. 47, D419-D426. doi: $10.1093 /$ nar/gky1038

Milet, C., and Monsoro-Burq, A. H. (2012). Neural crest induction at the neural plate border in vertebrates. Dev. Biol. 366, 22-33. doi: 10.1016/j.ydbio.2012.01. 013

Monsoro-Burq, A. H., Wang, E., and Harland, R. (2005). Msx1 and Pax3 cooperate to mediate FGF8 and WNT signals during Xenopus neural crest induction. Dev. Cell 8, 167-178. doi: 10.1016/j.devcel.2004.12.017

Nieto, M. A., Sargent, M. G., Wilkinson, D. G., and Cooke, J. (1994). Control of cell behavior during vertebrate development by Slug, a zinc finger gene. Science 264, 835-839.

Noden, D. M. (1975). An analysis of migratory behavior of avian cephalic neural crest cells. Dev. Biol. 42, 106-130.

Perez-Alcala, S., Nieto, M. A., and Barbas, J. A. (2004). LSox5 regulates RhoB expression in the neural tube and promotes generation of the neural crest. Development 131, 4455-4465. doi: 10.1242/dev.01329

Piacentino, M. L., and Bronner, M. E. (2018). Intracellular attenuation of BMP signaling via CKIP-1/Smurf1 is essential during neural crest induction. PLoS Biol. 16:e2004425. doi: 10.1371/journal.pbio.2004425

Piacentino, M. L., Hutchins, E. J., Andrews, C. J., and Bronner, M. E. (2020). Temporal changes in plasma membrane lipid content induce endocytosis to regulate developmental epithelial-to-mesenchymal transition. bioRxiv [Preprint]. doi: 10.1101/2020.10.18.344523

Rabadán, M. A., Herrera, A., Fanlo, L., Usieto, S., Carmona-Fontaine, C., Barriga, E. H., et al. (2016). Delamination of neural crest cells requires transient and reversible Wnt inhibition mediated by Dact1/2. Development 143, 2194-2205. doi: $10.1242 /$ dev.134981
Reid, B. S., Yang, H., Melvin, V. S., Taketo, M. M., and Williams, T. (2011). Ectodermal Wnt/beta-catenin signaling shapes the mouse face. Dev. Biol. 349, 261-269. doi: 10.1016/j.ydbio.2010.11.012

Ross, A. P., and Zarbalis, K. S. (2014). The emerging roles of ribosome biogenesis in craniofacial development. Front. Physiol. 5:26. doi: 10.3389/fphys.2014.00026

Schindelin, J., Arganda-Carreras, I., Frise, E., Kaynig, V., Longair, M., Pietzsch, T., et al. (2012). Fiji: an open-source platform for biological-image analysis. Nat. Methods 9, 676-682. doi: 10.1038/nmeth.2019

Simoes-Costa, M. S., McKeown, S. J., Tan-Cabugao, J., Sauka-Spengler, T., and Bronner, M. E. (2012). Dynamic and differential regulation of stem cell factor FoxD3 in the neural crest is Encrypted in the genome. PLoS Genet. 8:e1003142. doi: 10.1371/journal.pgen.1003142

Simoes-Costa, M., and Bronner, M. E. (2015). Establishing neural crest identity: a gene regulatory recipe. Development 142, 242-257. doi: 10.1242/dev.105445

Simoes-Costa, M., Stone, M., and Bronner, M. E. (2015). Axud1 Integrates Wnt signaling and transcriptional inputs to drive neural crest formation. Dev. Cell 34, 544-554. doi: 10.1016/j.devcel.2015.06.024

Steventon, B., Araya, C., Linker, C., Kuriyama, S., and Mayor, R. (2009). Differential requirements of BMP and Wnt signalling during gastrulation and neurulation define two steps in neural crest induction. Development 136, 771-779. doi: $10.1242 /$ dev.029017

Su, Y., Naser, I. B., Islam, S. M., Zhang, S., Ahmed, G., Chen, S., et al. (2009). Draxin, an axon guidance protein, affects chick trunk neural crest migration. Dev. Growth Differ. 51, 787-796. doi: 10.1111/j.1440-169X.2009.01137.x

Tahtakran, S. A., and Selleck, M. A. (2003). Ets-1 expression is associated with cranial neural crest migration and vasculogenesis in the chick embryo. Gene Expr. Patterns 3, 455-458.

Taneyhill, L. A., and Bronner-Fraser, M. (2005). Dynamic alterations in gene expression after Wnt-mediated induction of avian neural crest. Mol. Biol. Cell 16, 5283-5293. doi: 10.1091/mbc.e05-03-0210

Tawarayama, H., Yamada, H., Amin, R., Morita-Fujimura, Y., Cooper, H. M., Shinmyo, Y., et al. (2018). Draxin regulates hippocampal neurogenesis in the postnatal dentate gyrus by inhibiting DCC-induced apoptosis. Sci. Rep. 8:840. doi: 10.1038/s41598-018-19346-6

Theveneau, E., Duband, J. L., and Altabef, M. (2007). Ets-1 confers cranial features on neural crest delamination. PLoS One 2:e1142. doi: 10.1371/journal.pone. 0001142

Vega-Lopez, G. A., Cerrizuela, S., Tribulo, C., and Aybar, M. J. (2018). Neurocristopathies: new insights 150 years after the neural crest discovery. Dev. Biol. 444, S110-S143. doi: 10.1016/j.ydbio.2018.05.013

Wang, M. H., Sun, R., Zhou, X. M., Zhang, M. Y., Lu, J. B., Yang, Y., et al. (2018). Epithelial cell adhesion molecule overexpression regulates epithelialmesenchymal transition, stemness and metastasis of nasopharyngeal carcinoma cells via the PTEN/AKT/mTOR pathway. Cell Death Dis. 9:2. doi: 10.1038/ s41419-017-0013-8

Wu, J., Saint-Jeannet, J. P., and Klein, P. S. (2003). Wnt-frizzled signaling in neural crest formation. Trends Neurosci. 26, 40-45.

Wu, J., Yang, J., and Klein, P. S. (2005). Neural crest induction by the canonical Wnt pathway can be dissociated from anterior-posterior neural patterning in Xenopus. Dev. Biol. 279, 220-232. doi: 10.1016/j.ydbio.2004.12.016

Yanfeng, W., Saint-Jeannet, J. P., and Klein, P. S. (2003). Wnt-frizzled signaling in the induction and differentiation of the neural crest. Bioessays 25, 317-325. doi: 10.1002/bies. 10255

Zhang, S., Su, Y., Gao, J., Zhang, C., and Tanaka, H. (2017). A potential inhibitory function of draxin in regulating mouse trunk neural crest migration. In Vitro Cell. Dev. Biol. Anim. 53, 43-53. doi: 10.1007/s11626-0160079-0

Conflict of Interest: The authors declare that the research was conducted in the absence of any commercial or financial relationships that could be construed as a potential conflict of interest.

Copyright $($ C) 2021 Hutchins, Piacentino and Bronner. This is an open-access article distributed under the terms of the Creative Commons Attribution License (CC BY). The use, distribution or reproduction in other forums is permitted, provided the original author(s) and the copyright owner(s) are credited and that the original publication in this journal is cited, in accordance with accepted academic practice. No use, distribution or reproduction is permitted which does not comply with these terms. 